3. Philip Hartman and Aurel Wintner, On a comparison theorem for self-adjoint partial differential equations of elliptic type, Proc. Amer. Math. Soc. 6 (1955), 862-865.

4. Kurt Kreith, $A$ new proof of a comparison theorem for elliptic equations, Proc. Amer. Math. Soc. 14 (1963), 33-35.

5. Walter Leighton, Comparison theorems for linear differential equations of second order, Proc. Amer. Math. Soc. 13 (1962), 603-610.

6. H. A. Schwarz, Gesammelte Mathematische Abhandlungen, Vol. I, Springer, Berlin, 1890.

University of British Columbia, Vancouver, B. C., Canada

\title{
LOCAL TRAJECTORY EQUIVALENCE OF DIFFERENTIAL SYSTEMS
}

\section{COURTNEY COLEMAN ${ }^{1}$}

1. Consider the two differential systems

$$
d x / d t=f(x)
$$

and

$$
d x / d t=g(x),
$$

where $x$ is an $n$-vector, (1) and (2) each possess an asymptotically stable critical point which is taken without loss of generality to be the origin in each case, and $f$ and $g$ satisfy conditions sufficient to guarantee existence and uniqueness of solutions in some neighborhood of the origin. In any event, it is assumed that $f$ and $g$ are continuous near the origin. In general, systems (1) and (2) are said to be locally trajectory equivalent with respect to the regions $R$ and $S$ if there is a homeomorphism of $R$ onto $S$ mapping arcs of trajectories of (1) in $R$ onto arcs of trajectories of (2) in $S$. In this note the following theorem is proved.

THEOREM. Under the conditions on $f$ and $g$ given above, there is a pair of neighborhoods of the origin with respect to which systems (1) and (2) are locally trajectory equivalent.

Received by the editors August 24, 1964.

1 This research was supported in part by the United States Air Force through the Air Force Office of Scientific Research, Office of Aerospace Research, under Contract No. AF 49(638)-1242, in part by the National Aeronautics and Space Administration under Contract No. NASw-8411, in part by the Office of Naval Research under Contract No. Nonr-3693(00), and in part by the National Science Foundation under Grant G-23664. 
In other words, all asymptotically stable critical points can be identified up to local trajectory equivalence. Of course, the same is true for asymptotically unstable critical points. The proof of this theorem is easy, although the theorem itself seems to be new. In fact, Minc [1] proved in a quite complicated way a similar theorem for the case $n=3$, where the critical points were (geometrically) simple nodes or node-foci. No such assumptions about the geometric nature of the critical points are needed here, nor is $n$ restricted. Other theorems on trajectory equivalence have depended upon assumptions on the linear approximations to $f$ and $g$ at the origin. For instance, if $f$ and $g$ are $C^{1}$ at the origin and if the coefficient matrices of their linear approximations have the same number of roots with negative real parts and have no roots with zero real parts, then it can be shown that (1) and (2) are locally trajectory equivalent with respect to certain neighborhoods of the origin [2]. One can obtain local trajectory equivalence via a $C^{1}$ homeomorphism if $g(x)=A x$ is the linear approximation to $f(x)$ and if the real parts of the roots of the matrix $A$ are all of the same sign and none have zero real part [3]. Here, we only require that the coefficient matrices of the linear approximations not have roots whose real parts are positive, for there would be no asymptotic stability in that event. The linear approximations may, in fact, vanish. In general, it is not possible to define a $C^{1}$-homeomorphism, since no assumptions are made concerning the modes of approach of trajectories to the origin.

2. The proof rests upon the following result of Massera [4]: if the origin is an asymptotically stable critical point of (1), then there exists a $C^{\infty}$ function $V_{1}(x)$ which is positive definite with negative definite derivative $d V_{1}(x) / d t$ computed along the trajectories of (1). For small enough positive $c$ the surface defined by $V_{1}(x)=c$ is a diffeomorph of the unit $n-1$ sphere, contains the origin in its interior and is a surface of section relative to the flow defined by (1), trajectories of (1) crossing the surface into its interior with increasing $t$. Let $\Sigma_{1}$ denote a particular one of these surfaces. Since $d V_{1}(x) / d t$ is negative definite, every trajectory, except for the origin, with points in $B_{1}$ (the union of $\Sigma_{1}$ and its interior) cuts $\Sigma_{1}$ exactly once and approaches the origin with increasing $t . V_{2}, \Sigma_{2}$ and $B_{2}$ can be defined similarly relative to system (2).

Let $\phi$ be any map of $\Sigma_{1}$ onto $\Sigma_{2}$ which is a homeomorphism in the relative topology. If $p$ is a point of $B_{1}$, define $\eta(p)$ to be the origin if $p$ is, otherwise to be $x^{2}\left(\phi\left(x^{1}\left(p,-t_{p}\right)\right), t_{p}\right)$, where $x^{i}(q, t), i=1,2$, denotes the solution of system (i) for which $q=x^{i}(q, 0)$ and where $t_{p}$ is 
the unique non-negative number such that $x^{1}\left(p,-t_{p}\right)$ defines a point of $\Sigma_{1}$. The domain of $\eta$ is $B_{1}$, its range is a subset of $B_{2}$, it is $1: 1$ and it is continuous on $B_{1}-\{0\}$. In addition, $\eta$ maps arcs of trajectories of (1) in $B_{1}$ onto arcs of trajectories of (2) in $B_{2}$. The theorem will be proved if it can be shown that $\eta$ is continuous at the origin, for then the range of $\eta$ will be $B_{2}$ and $R$ can be taken to be $B_{1}$ and $S$ to be $B_{2}$, $R$ and $S$ the neighborhoods in the definition of local trajectory equivalence.

To show that $\eta$ is continuous at the origin, we first note that there can be no sequence of points $\left\{p_{n}\right\}$ of $B_{1}$ converging to the origin but such that $t_{p_{n}}$ converges to some finite limit $T$. If there were such a sequence, there would be a subsequence $\left\{p_{n_{j}}\right\}$ such that $x^{1}\left(p_{n_{j}},-t_{p_{n_{j}}}\right)$ converges to a point $q$ of $\Sigma_{1}$. But then $x^{1}(q, T)=0$, which is impossible. Thus, as $p \rightarrow 0, t_{p} \rightarrow \infty$ and $x^{2}\left(\phi\left(x^{1}\left(p,-t_{p}\right), t_{p}\right)\right) \rightarrow 0$ - hence $\eta$ is continuous at 0 .

\section{REFERENCES}

1. R. Minc, $O$ topologičeskot ekvivalentnosti sostoyanit ravnovesiya sistemy treh differencial' nyh uravnenił, Naux̌. Dokl. Vysక̌. Školy, Fiz.-Mat. Nauki (1958), no. 1, 19-24.

2. D. Grobman, Topologičeskaya klassifikacia osobor točki v n-mernom prostranstve, Mat. Sb. (N.S.) 56 (98) (1962), 77-94.

3. P. Hartman, On local homeomorphisms of Euclidean spaces, Bol. Soc. Mat. Mexicana (2) 5 (1960), 220-241.

4. J. Massera, Contributions to stability theory, Ann. of Math. 64 (1956), 182-206.

RIAS, BALTIMORE AND

Harvey Mudd College 